tsyfrovykh system supervyzornoho upravlenyia kolonnoi rektyfykatsyy nefty", Avtomatyzatsyia tekhnolohycheskykh y byznes - protsessov, vol. 7, no. 4, pp. 24-33, 2015.

[9] A. Stopakevych, Systemnyi analyz y teoryia slozhnykh system upravlenyia. Odessa: Astroprynt, 2013.

[10] L. Szabó, "Three-Level Control of a Distillation Column," Engineering, vol. 04, no. 10, pp. 675-681, 2012.

\title{
АНАЛИЗ ПЕРЕДАТОЧНЫХ ФУНКЦИЙ ЦИФРОВЫХ ЧАСТОТНО-ЗАВИСИМЫХ КОМПОНЕНТ НИЗКОГО ПОРЯДКА
}

\author{
А. В. Ухина ${ }^{1}$ \\ ${ }^{1}$ Odessa national polytechnical university, Ukraine \\ ${ }^{1}$ ORCID: 0000-0003-3797-1460 \\ E-mail: ${ }^{1}$ anyuta.uhina@inbox.ru
}

Copyright (C) 2014 by author and the journal -Automation technological and business - processes\|. This work is licensed under the Creative Commons Attribution International License (CC BY). http://creativecommons.org/licenses/by/4.0/

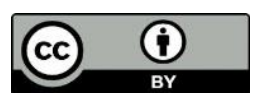

ONAFT

Open Access

Аннотация: При проектировании компонент специализированных компьютерных систем возникают задачи, связанные с уменьшением объема вычислений при проектировании и ускорении процесса настройки. В работе проведен анализ и получены соотночения коэффициентов передаточных функций цифрровых частотно-зависимых компонент первого, второго и четвертого порядков, что позволяет упростить проиесс проектирования.

Abstract: When designing components of specialized computer systems the takes related to the reduction in the amount of computation arise during the design and acceleration of the tuning process. To solve these problems, an analysis was made and algorithms for calculating generalized transfer functions analog components of the first and second order were determined, which made it possible to solve the given problems

Ключевые слова: Частотно-зависимый компонент, нормированный фильтр, коэффициенты числителя и знаменателя, частота среза, центральная частота.

Key-words: Frequency-dependent component, normalized filter, coefficients of the numerator and denominator, cutoff frequency, center frequency.

Частотно-зависимые компоненты (ЧЗК) широко используются в трактах обработки сигналов в специализированных компьютерных системах. При проектировании подобных компонент возникает необходимость ускорить процесс проектирования и уменьшить объем вычислений, а при настройке тракта уменьшить и это время.

Аналогичные задачи возникали и при проектировании аналоговых компонент [1-3]. Для решения этой задачи был проведен анализ обобщенных передаточных функций первого и второго порядков аналоговых компонент

$$
K_{1}(\mathrm{p})=\mathrm{k}_{01} \frac{a+b p}{c+d p}, K_{2}(\mathrm{p})=\mathrm{k}_{02} \frac{a+b p+e p^{2}}{c+d p+f p^{2}}
$$

и для них определены алгоритмы расчета. В работе [4] приведены передаточные функции и алгоритмы расчета для аналоговых частотно-зависимых компонент низкого порядка с комплексными корнями знаменателя. Такие передаточные функции соответствуют фильтрам и их можно представить в виде

- фильтра нижних частот (ФНЧ) - $K(\mathrm{p})=\mathrm{k}_{0} \frac{1}{1+a p}$; 
- фильтра верхних частот (ФВЧ) - $K(\mathrm{p})=\mathrm{k}_{0} \frac{p}{1+a p}$;

- полосового фильтра (ПФ) - $K(\mathrm{p})=\mathrm{k}_{0} \frac{B p}{A+B p+p^{2}}$;

- режекторного фильтра (РФ) - $K(\mathrm{p})=\mathrm{k}_{0} \frac{A+p^{2}}{A+B p+p^{2}}$;

Такое представление позволило сократить объем вычислений на этапе проектирования. Анализ ЧЗК низкого порядка связан с тем, что проектирование перестраиваемых компонент высокого порядка основано на соединении компонент низкого порядка [4-6], что облегчает проектирование и перестройку (настройку) тракта обработки сигналов.

При переходе к цифровым частотно-зависимым компонентам обобщенные передаточные функции первого и второго порядков имеют вид [7]

$$
\begin{gathered}
H_{1}(\mathrm{z})=\frac{a_{0}+a_{1} z^{-1}}{1+b_{1} z^{-1}} ; \\
H_{2}(\mathrm{z})=\frac{a_{0}+a_{1} z^{-1}+a_{2} z^{-2}}{1+b_{1} z^{-1}+b_{2} z^{-2}},
\end{gathered}
$$

что затрудняет у разработчиков выполнения расчетов. В этом случае возникает необходимость уменьшить число рассчитываемых и настраиваемых коэффициентов, а также задача проведения анализа вида передаточных функций цифровых ЧЗК низкого порядка.

Исследованы нормированные цифровые ЧЗК компоненты первого, второго и четвертого порядков ФНЧ, ФВЧ, ПФ, РФ типов Баттерворта, Чебышева, инверсного Чебышева и эллиптического. При изменении относительной частоты среза $\bar{\omega}$ для ФНЧ и ФВЧ на отрезке $\bar{\omega}=[0.1 \div 0.9]$ и относительной центральной частоты $\bar{\omega}_{0}$ для ПФ и РФ на отрезке $\overline{\omega_{0}}=[0.2 \div 0.8]$, при этом $\bar{\omega}_{0}=\frac{\left(\overline{\omega_{1}}+\overline{\omega_{2}}\right)}{2}, \bar{\omega}_{1}=\bar{\omega}_{0}-0.1, \bar{\omega}_{2}=\bar{\omega}_{0}+0.1$ и при изменении уровня колебательности -0.5 дБ, 3 дБ, -20 дБ, где $\bar{\omega}=\frac{2 \pi \frac{f}{f_{d}}}{\pi}, \bar{\omega} \in[0 \div 1], f, f_{d}$ - соответственно текущая частота и частота дискретизации.

1. Частотно-зависимый компонент первого порядка

Передаточная функция ЧЗК первого порядка имеет вид (1). При этом ФНЧ соответствует передаточная функция при коэффициенте числителя $a_{1} \triangleright 0$, а ФВЧ $-a_{1} \triangleleft 0$.

Следует отметить, что коэффициенты числителя нормированных цифровых ЧЗК первого порядка равны, т.е. для ФНЧ

а для ФВЧ

$$
a_{0}=a_{1},
$$

$$
a_{0}=-a_{1}
$$

При учете (3) и (4) передаточную функцию (1) можно записать в виде

$$
H(\mathrm{z})=a_{0} \frac{1 \pm \frac{a_{1}}{a_{0}} z^{-1}}{1+b z^{-1}}=a_{0} \frac{1 \pm z^{-1}}{1+b z^{-1}}
$$

где знак «+» в числителе соответствует ФНЧ, а знак «-» - ФВЧ. При этом коэффициенты ЧЗК Баттерворта равны коэффициентам ЧЗК Чебышева, инверсного Чебышева и эллиптического соответственно.

Также при увеличении частоты среза $\bar{\omega} \uparrow$ коэффициенты числителя и знаменателя ФНЧ возрастают, т.е. $b \uparrow, a_{0} \uparrow$, а ФВЧ $-b \uparrow, a_{0} \downarrow$.

Для ЧЗК ПФ и РФ первого порядка описываются передаточными функциями второго порядка, поэтому далее рассмотрим ЧЗК второго порядка.

2. Частотно-зависимый компонент второго порядка

Передаточная функция второго порядка имеет вид (2).

2.1. Фильтры нижних и верхних частот

В ходе исследований было выявлено, что два коэффициента числителя равны:

$$
a_{0}=a_{2},
$$

С учетом (6) передаточную функцию (2) можно записать в виде 


\section{http://atbp.onaft.edu.ua/}

$$
H(\mathrm{z})=a_{0} \frac{1+\frac{a_{1}}{a_{0}} z^{-1}+z^{-2}}{1+b_{1} z^{-1}+b_{2} z^{-1}}=a_{0} \frac{1+a z^{-1}+z^{-2}}{1+b_{1} z^{-1}+b_{2} z^{-2}},
$$

для ФНЧ Баттерворта и Чебышева коэффициент числителя $a=\frac{a_{1}}{a_{0}}=2$, а для ФВЧ - $a=-2$, т.к. $a_{1} \triangleleft 0$.

При увеличении частоты $\bar{\omega} \uparrow$ коэффициент знаменателя возрастает $b_{1} \uparrow$, а $b_{2}-$ до середины диапазона частоты уменьшается, а затем снова возрастает, т.е. $b_{2} \downarrow \in\left[\overline{\omega_{\min }}, \overline{\omega_{c p}}\right) \cup b_{2} \uparrow \in\left(\overline{\omega_{c p}}, \overline{\omega_{\max }}\right]$. Коэффициенты числителя возрастают $a_{0}=a_{2} \uparrow, a_{1} \uparrow$ для ФНЧ, а для ФВЧ $-a_{0}=a_{2} \downarrow, a_{1} \uparrow$.

2.2. Полосовые и режекторные фильтры

Коэффициенты числителя полосовых и режекторных фильтров, описанных передаточной функцией (2) равны (6). Однако, для ПФ коэффициент $a_{2} \triangleleft 0$ и $a_{1}=0$, поэтому уравнение (7) примет вид

$$
H(\mathrm{z})=a_{0} \frac{1-z^{-2}}{1+b_{1} z^{-1}+b_{2} z^{-2}},
$$

а РФ будет соответствовать уравнение (7).

При увеличении центральной частоты $\bar{\omega}_{0} \uparrow$ коэффициенты знаменателя $b_{2}$ и числителя $a_{0}=\left|a_{2}\right|$ остаются неизменными, $\quad$ т.е. $\quad b_{2}\left(\overline{\omega_{0}}\right)=$ const,$a_{0}\left(\overline{\omega_{0}}\right)=\left|a_{2}\left(\overline{\omega_{0}}\right)\right|=$ const . Коэффициент $\quad$ знаменателя $\quad b_{1} \quad$ изменяется $\quad$ как $b_{1} \downarrow \in\left[\overline{\omega_{0 \min }}, \overline{\omega_{0 c p}}\right) \cup b_{1} \uparrow \in\left(\overline{\omega_{0 c p}}, \overline{\omega_{0 \max }}\right] \cup b_{1}\left(\overline{\omega_{0 c p}}\right)=0 ; b_{1} \in\left[\overline{\omega_{0 \min }}, \overline{\omega_{0 c p}}\right)=-b_{1} \in\left(\overline{\omega_{0 c p}}, \overline{\omega_{0 \max }}\right]$. Следует отметить, что для РФ коэффициенты $b_{1}=a_{1}$.

3. Частотно-зависимый компонент четвертого порядка

Частотно-зависимый компонент четвертого порядка описывается уравнением

$$
H(\mathrm{z})=\frac{a_{0}+a_{1} z^{-1}+a_{2} z^{-2}+a_{3} z^{-3}+a_{4} z^{-4}}{1+b_{1} z^{-1}+b_{2} z^{-2}+b_{3} z^{-3}+b_{4} z^{-4}},
$$

где $a_{0}, a_{1}, a_{2}, a_{3}, a_{4}$ - действительные коэффициенты числителя; $b_{1}, b_{2}, b_{3}, b_{4}$ - действительные коэффициенты знаменателя.

\section{1. Фильтры нижних и верхних частот}

Коэффициенты числителя уравнения (9) соотносятся как

Тогда уравнение (9) можно записать, как

$$
a_{0}=a_{4}, a_{1}=a_{3}
$$

$$
H(\mathrm{z})=a_{0} \frac{\frac{a_{1}}{a_{0}} z^{-1}+\frac{a_{2}}{a_{0}} z^{-2}+\frac{a_{3}}{a_{0}} z^{-3}+z^{-4}}{1+b_{1} z^{-1}+b_{2} z^{-2}+b_{3} z^{-3}+b_{4} z^{-4}}=a_{0} \frac{A_{1}\left(\mathrm{z}^{-1}+\mathrm{z}^{-3}\right)+\mathrm{A}_{2} z^{-2}+z^{-4}}{1+b_{1} z^{-1}+b_{2} z^{-2}+b_{3} z^{-3}+b_{4} z^{-4}},
$$

где $A_{1}=\frac{a_{1}}{a_{0}}=\frac{a_{3}}{a_{0}}, A_{2}=\frac{a_{2}}{a_{0}}$.

Для ФНЧ Баттерворта и Чебышева $A_{1}=4, A_{2}=6$, для ФВЧ $-A_{1}=-4, A_{2}=6$.

При изменении частоты среза $\bar{\omega} \uparrow$ коэффициенты знаменателя возрастают $b_{1}, b_{3} \uparrow$, a $b_{2}, b_{4}$ - до середины диапазона частоты уменьшаются, а затем снова возрастает, $b_{2}, b_{4} \downarrow \in\left[\overline{\omega_{\min }}, \overline{\omega_{c p}}\right) \cup b_{2}, b_{4} \uparrow \in\left(\overline{\omega_{c p}}, \overline{\omega_{\max }}\right]$. Коэффициенты числителя возрастают $a_{0}=a_{4} \uparrow, a_{1}=a_{3} \uparrow$ для ФНЧ, а для ФВЧ - $a_{0}=a_{4} \downarrow, a_{1}=a_{3} \uparrow$.

3.2. Полосовые и режекторные фильтры

Эти фильтры второго порядка описывается уравнением четвертого порядка (9) и коэффициенты числителя соотносятся как (10). Однако у ПФ Баттерворта и Чебышева коэффициенты числителя $a_{1}=a_{3}=0$ и уравнение (11) примет вид

$$
H(\mathrm{z})=a_{0} \frac{A_{2} z^{-2}+z^{-4}}{1+b_{1} z^{-1}+b_{2} z^{-2}+b_{3} z^{-3}+b_{4} z^{-4}}
$$


Так же следует отметить, что коэффициенты числителя $a_{0}\left(\overline{\omega_{0}}\right)=a_{4}\left(\overline{\omega_{0}}\right)=$ const и коэффициент знаменателя $b_{4}\left(\overline{\omega_{0}}\right)=$ const. При изменении центральной частоты $\overline{\omega_{0}} \uparrow$ коэффициенты знаменателя $b_{1}$ и $b_{3}$ изменяются как $b_{1}, b_{3} \downarrow \in\left[\overline{\omega_{0 \min }}, \overline{\omega_{0 c p}}\right) \cup b_{1}, b_{3} \uparrow \in\left(\overline{\omega_{0 c p}}, \overline{\omega_{0 \max }}\right]$. В ходе анализа были выявлены следующие закономерности

$$
\left\{\begin{array}{l}
b_{1}, b_{3} \in\left[\overline{\omega_{0 \min }}, \overline{\omega_{0 c p}}\right)=-b_{1}, b_{3} \in\left(\overline{\omega_{0 c p}}, \overline{\omega_{0 \max }}\right] \\
b_{1}, b_{3}\left(\overline{\omega_{0 c p}}\right)=0 ; \\
b_{2} \in\left[\overline{\omega_{0 \min }}, \overline{\omega_{0 c p}}\right)=b_{2} \in\left(\overline{\omega_{0 c p}}, \overline{\omega_{0 \max }}\right] \\
a_{1}, a_{3} \in\left[\overline{\omega_{0 \min }}, \overline{\omega_{0 c p}}\right)=-a_{1}, a_{3} \in\left(\overline{\omega_{0 c p}}, \overline{\omega_{0 \max }}\right] \\
a_{1}, a_{3}\left(\overline{\omega_{0 c p}}\right)=0 ; \\
a_{2} \in\left[\overline{\omega_{0 \min }}, \overline{\omega_{0 c p}}\right)=a_{2} \in\left(\overline{\omega_{0 c p}}, \overline{\omega_{0 \max }}\right]
\end{array}\right.
$$

Таким образом, проведенный анализ позволяет сократить число вычисляемых коэффициентов, а полученные соотношения и закономерности в поведении коэффициентов поможет в настройке трактов обработки входных сигналов в специализированных компьютерных системах. Уменьшения количества коэффициентов в передаточных функциях упрощает алгоритмы их вычисления и объемов вычислений, что сокращает, в общем, процесс проектирования.

\section{Литература}

[1] Гаврилов Л. П. Расчет и моделирование линейных электрических цепей с применением ПК / Л. П. Гаврилов, Д. А. Соснин. - М.: СОЛОН-Пресс, 2004. - 448 с.

[2] Карлащук В. И. Электронная лаборатория на ІВМРС. Моделирование элементов аналоговых систем / В. И. Карлащук. - М.: СОЛОН-Пресс, 2006. - Т.1. - 672 с.

[3] Малахов В. П. Анализ основных свойств матриц аналоговых и цифровых устройств / В. П. Малахов, В. С. Ситников, П. В. Ступень // Теоретический и научно-практический журнал по техническим и естественным наукам : сб. труд. / Одесск. нац. политех. ун-т. - Одесса, 1996. - №2 Автоматика и системотехника. Радиоэлектроника. Гуманитарные науки. - С. 5-6.

[4] Мошиц Г. Проектирование активных фильтров / Г. Мошиц, П. Хорн. - М.: Мир, 1984. - 320 с.

[5] Ситников В. С. Реализация цифрового фильтра высокого порядка в каскадной форме по критерию минимума выходного шума квантования. // Автоматизация. Электротехнические комплексы и системы (ААЭКС) / В. С. Ситников. - Херсон, 2003. - №2 (12). - С. 100-108.

[6] Ситніков В. С. Вплив особливостей каскадів цифрового фільтру високого порядку на вихідний шум квантування. // Электротехнические и компьютерные системы / В. С. Ситніков, П. В. Ступень, Б. О. Півень. 2013. - №9 (85). - C. 97-101.

[7] Stoyanov G. Design of variable IIR digital filters using equal subfilters / G. Stoyanov, I. Uzunov, M. Kawamata // Intelligent Signal Processing and Communication Systems, Proceedings of IEEE International Symposium. - IEEE, 2000. - T. 1. - C. 141-146.

\section{References}

[1] L. P. Gavrilov and D. A. Sosnin, Calculation and modeling of linear electric circuits using a PC. Moscow: SOLONPress Publ., 2004;

[2] V. I. Karlaschuk, Electronic laboratory on IBMPC. Tom 1. Modeling of elements of analog systems. Moscow: SOLON-Press Publ., 2006;

[3] V. P. Malakhov, et al., "Analysis of the basic properties of matrices of analog and digital devices," Trudy Odesskogo politekhnicheskogo universiteta, no. 2, pp. 5-6, 1996;

[4] G. Moshits and Khorn M., Designing active filters. Moscow, 1984;

[5] V. S. Sitnikov, "Designing active filters the implementation of a high-order digital filter in cascade form by the criterion of a minimum of quantization output noise," Avtomayozatsiya. Elektricheskie kompleksy i sistemy, no. 2(12), pp. 100108, 2003;

[6] V. S. Sytnikov, et al., "Impact features a digital filter stages to output high-order quantization noise," Elektricheskie $i$ kimputernye sistemy, no.9(85), pp. 97-101, 2013;

[7] G. Stoyanov, et al., "Design of variable IIR digital filters using equal subfilters," Proceedings of IEEE International Symposium on Intelligent Signal Processing and Communication Systems, vol. 1, pp. 141-146, 2000. 